\title{
Acute Kidney Injury and Long-Term Risk for Cardiovascular Events in Patients after Kidney Transplantation
}

\author{
Ruth Rahamimov ${ }^{a-c}$ Tuvia Y. van Dijk ${ }^{c, d}$ Maya Molchoc Itay Lahav ${ }^{c}$ \\ Eytan Mor $^{b, c}$ Naomy Ben Dor ${ }^{a, c}$ Shira Goldman ${ }^{a, c}$ Benaya Rozen-Zvi ${ }^{a, c}$ \\ a Department of Nephrology and Hypertension, Rabin Medical Center, Beilinson Campus, \\ Petah-Tikva, Israel; ${ }^{b}$ Department of Transplantation, Rabin Medical Center, Beilinson \\ Campus, Petah-Tikva, Israel; ' Sackler Faculty of Medicine, Tel Aviv University, Tel Aviv, Israel; \\ dDepartment of Emergency Medicine, Rabin Medical Center, Beilinson Campus, \\ Petah-Tikva, Israel
}

\section{Keywords}

Acute kidney injury · Major adverse cardiovascular events · Kidney transplantation

\begin{abstract}
Background: Acute kidney injury (AKI) was found to be associated with an increased risk of major adverse cardiovascular events (MACE) in the general population. Patients after kidney transplantation are prone to AKI events and are also at an increased risk of cardiovascular (CV) disease. The association between AKI and MACE in kidney transplant patients is yet to be studied. Methods: This retrospective single-center cohort study reviewed 416 adult renal allograft recipients transplanted between 2005 and 2010. AKI events were recorded starting 2 weeks after transplantation, or following discharge with a functioning graft. AKI was defined, according to the KDIGO criteria. The primary outcome was the composite of MACE starting 6 months after transplantation and all-cause mortality. For survival analysis, we used univariate and multivariate time varying Cox proportional hazard model. Results: One hundred and twenty-four patients (29.8\%) had at least one episode of AKI. During the median follow-up time of 7.2 years (interquartile range 4.3-9.1), 144 outcome events occurred. By time varying Cox regression analysis, AKI was associated with an increased rate of CV outcomes or death (hazard ratio [HR] $1.96,95 \% \mathrm{Cl} 1.36-2.81, p<0.001)$, and the association remained significant by multivariate adjusted model (HR 1.76,95\% Cl 1.18-2.63, $p=0.005$ ). As for the different components of MACE, all-cause mortality and CV mortality were the only outcomes that were significantly associated with AKI. No interaction between AKI timing and MACE was found. Conclusion: AKI in kidney transplant recipient is associated with an increased risk of CV disease.
\end{abstract}




\section{Kidney \\ Blood Pressure \\ Research}

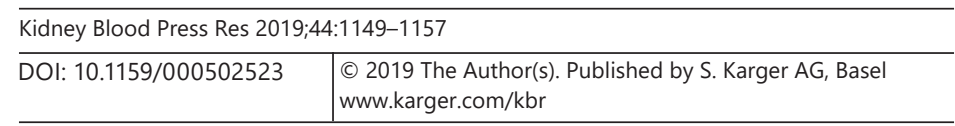

Rahamimov et al.: AKI and CV Events after Kidney Transplantation

\section{Introduction}

Patients after kidney transplantation are susceptible to acute kidney injury (AKI) events, with a reported incidence of between 11.3 and 51.14\% depending on the timing, duration of follow-up, and AKI definition [1-6]. AKI was reported in up to $82.3 \%$ of hospital admissions of patients after kidney transplantation [4]. This high prevalence of AKI in kidney recipients is due to standard risk factors as well as risk factors unique to this population such as a single functioning kidney, chronic exposure to nephrotoxic medications, susceptibility to infection, and the risk of acute rejection [1-8]. AKI after kidney transplantation was associated with an increased risk of long-term graft dysfunction or loss [1-6, 8] and death from all causes [3].

Patients after kidney transplantation are also at an increased risk of major adverse cardiovascular events (MACE) due to the combination of a higher prevalence of traditional risk factors such as hypertension, diabetes, dyslipidemia, and obesity as well as non-traditional risk factors such as renal impairment, anemia, proteinuria, and left ventricular hypertrophy [9-11].

A recent meta-analysis of AKI in the general population reported that AKI is associated with an increased risk of cardiovascular (CV) mortality and MACEs, particularly heart failure and acute myocardial infarction [12]. The association between AKI and CV events in patients after kidney transplantation has not been investigated yet. In this study, we intend to evaluate whether there is an association between AKI events and MACE in patients after kidney transplantation.

\section{Patients and Methods}

The study cohort included patients who underwent kidney transplantation between January 2005 and December 2010. Patients with multiple organ transplantations and patients with graft survival of $<6$ months were excluded. As MACE occurring in the immediate posttransplantation period can be the result of pre-transplantation and procedure-related factors, we excluded patients that had MACE during the first 6 months after transplantation.

The study was approved by our Institutional Review Board and was carried out in line with the Declarations of Helsinki and Istanbul. As this is a retrospective study, informed consent was not needed.

\section{Exposure}

Follow-up began 2 weeks after transplantation, or after discharge with a functioning graft. AKI was defined, according to the KDIGO criteria, as an elevation in serum creatinine (SCr) of $50 \%$ or more from baseline. Baseline $\mathrm{SCr}$ was defined as the average of the $2 \mathrm{SCr}$ tests before the index measurement. All available SCr measurements were screened automatically, and every increase of $50 \%$ and more from baseline was evaluated by a study researcher (T.Y.D.) who reviewed the medical records. In unclear cases, the data were evaluated by another researcher (B.R.-Z.), and in case of disagreement regarding the diagnosis of AKI, another researcher (R.R.) examined the data and made a decision. We collected all AKI events during the first 5 years after the transplantation or end of follow-up. For each episode we collected the timing, peak SCr level, and recovery (SCr within $20 \%$ of baseline value after 1 month from the index measurement).

\section{Outcomes}

The primary outcome was the composite of MACE and all-cause mortality. We included all-cause mortality for a number of reasons. First, the etiology of death is, usually, multifactorial and the contribution of CV disease might be underappreciated. Second, the recorded 


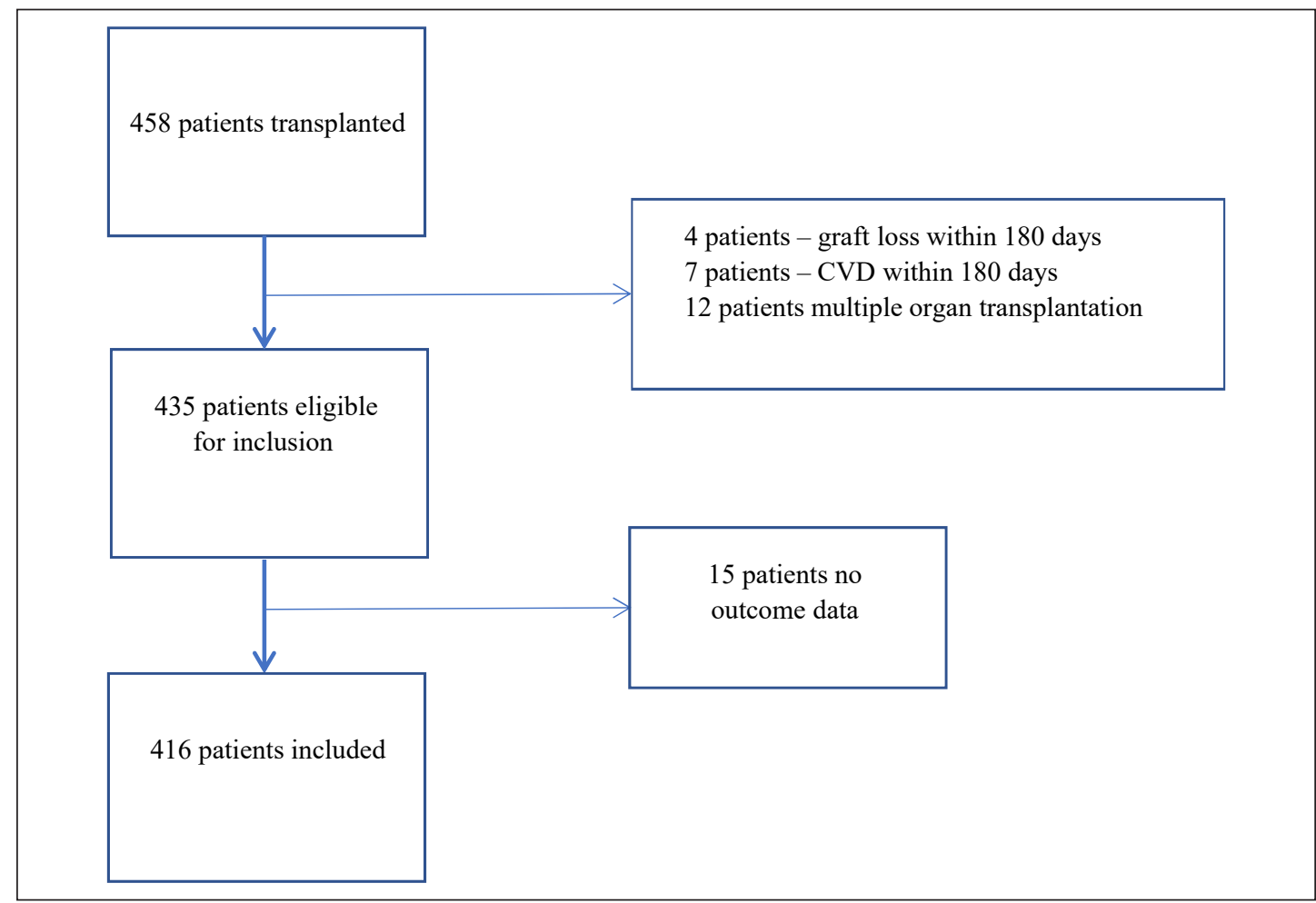

Fig. 1. Flow chart of patient's inclusion into the study. CVD, cardiovascular disease.

cause of death might be misleading and thirdly, including all-cause mortality in the composite outcome reduces the risk of bias by competing risks. The secondary outcomes included death censored MACE (including CV death and censoring death attributed to other etiologies) and the individual CV outcomes; coronary artery disease (CAD), cerebrovascular disease, peripheral vascular disease (PVD), and CV mortality.

CAD was defined as myocardial infraction, acute coronary syndrome, and coronary artery revascularization (percutaneously or by surgery). Cerebrovascular disease included cerebrovascular accident (CVA), transient ischemic attack or carotid or cerebral revascularization. PVD was defined as hospitalization due to peripheral ischemia, need for revascularization or need for amputation due to limb ischemia. Congestive heart failure (CHF) was defined according to an echocardiogram demonstrating new onset reduced systolic heart function or hospitalization due to pulmonary or peripheral edema attributed to cardiac origin. CV mortality was defined as mortality attributed to CVA, PVD, CAD arrhythmia or CHF.

All outcomes were collected from our electronic medical records system that provides a detailed report of all the hospitalizations during the study period, including other hospitals in Israel. Two study researchers (M.M. and I.V.) evaluated all reports for outcome events and, in case of any doubt, another researcher (B.R.-Z.) evaluated the report and made the final decision.

\section{Statistical Analysis}

Results are expressed as mean and SD for normally distributed data and median and interquartile range (IQR) for non-normally distributed data. Differences between the means of different groups were analyzed using Student $t$ test for normally distributed and MannWhitney U test for non-normally distributed data. Differences in frequency distribution were estimated using the $\chi^{2}$ test. 


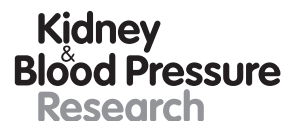

\begin{tabular}{l|l}
\hline \multicolumn{2}{l}{ Kidney Blood Press Res 2019;44:1149-1157 } \\
\hline DOI: 10.1159/000502523 & $\begin{array}{l}\text { (c) 2019 The Author(s). Published by S. Karger AG, Basel } \\
\text { www.karger.com/kbr }\end{array}$ \\
\hline
\end{tabular}

Rahamimov et al.: AKI and CV Events after Kidney Transplantation

Table 1. Comparison between patients with and without AKI

\begin{tabular}{|c|c|c|c|c|c|}
\hline & $n(\%)$ & All (416) & AKI (124) & No AKI (292) & $p$ value \\
\hline Men, $n(\%)$ & $416(100.0)$ & 267 (64.2) & $78(62.9)$ & $189(64.7)$ & 0.72 \\
\hline Age, years, mean \pm SD & $416(100.0)$ & $47.5 \pm 15.3$ & $45.6 \pm 16.2$ & $48.3 \pm 14.8$ & 0.11 \\
\hline Diabetes, $n(\%)$ & $416(100.0)$ & $112(26.9)$ & $34(27.4)$ & $78(26.4)$ & 0.88 \\
\hline $\mathrm{HD}, n(\%)$ & $416(100.0)$ & $82(19.7)$ & $30(24.2)$ & $52(17.8)$ & 0.13 \\
\hline HTN, $n(\%)$ & $324(77.9)$ & $218(67.3)$ & $82(74.5)$ & $136(63.6)$ & 0.046 \\
\hline Living donor, $n(\%)$ & $416(100.0)$ & $195(46.9)$ & $66(53.2)$ & $129(44.2)$ & 0.091 \\
\hline Donor age, years, mean \pm SD & $416(100.0)$ & $43.8 \pm 14.3$ & $43.7 \pm 15$ & $44.2 \pm 14.5$ & 0.72 \\
\hline Smoking, $n(\%)$ & $370(88.9)$ & $68(18.4)$ & $27(24.3)$ & $41(15.58)$ & 0.053 \\
\hline PRA $\geq 10 \%$ & $416(100.0)$ & $48(11.5)$ & $16(12.9)$ & $32(11.0)$ & 0.57 \\
\hline DGF & $404(97.1)$ & 107 (26.5) & $46(38.7)$ & $61(21.4)$ & $<0.001$ \\
\hline Hospital stay & $416(100.0)$ & $14.5 \pm 8.4$ & $15.5 \pm 9$ & $14.1 \pm 8.1$ & 0.1 \\
\hline Induction & $416(100.0)$ & & & & 0.14 \\
\hline Il-2 antagonists & & $272(65.4)$ & $82(66.1)$ & $190(65.1)$ & \\
\hline Thymoglobulin & & $58(13.9)$ & $16(12.9)$ & $42(14.3)$ & \\
\hline Other & & $12(2.9)$ & $7(5.6)$ & $5(1.7)$ & \\
\hline None & & $74(17.8)$ & $19(15.3)$ & $55(18.8)$ & \\
\hline \multicolumn{6}{|l|}{ Medications } \\
\hline $\mathrm{CCB}, n(\%)$ & $324(77.9)$ & $135(41.7)$ & $52(47.3)$ & $83(38.8)$ & 0.14 \\
\hline Beta blockers, $n(\%)$ & $325(78.1)$ & $161(49.5)$ & $59(53.6)$ & $102(47.4)$ & 0.29 \\
\hline Statins, $n(\%)$ & $325(78.1)$ & $152(46.8)$ & $45(40.9)$ & $107(49.8)$ & 0.13 \\
\hline Anti-aggregant & $325(78.1)$ & $258(79.4)$ & $89(80.9)$ & $169(78.6)$ & 0.63 \\
\hline ACEI/ARB, $n(\%)$ & $324(77.9)$ & $62(21.6)$ & $22(20.0)$ & $40(22.4)$ & 0.62 \\
\hline Immunosuppression & $411(98.8)$ & & & & 0.31 \\
\hline Tacrolimus, $n(\%)$ & & $372(79.4)$ & $108(88.5)$ & $264(90.3)$ & \\
\hline Cyclosporine, $n(\%)$ & & $23(5.6)$ & $10(8.2)$ & $13(4.5)$ & \\
\hline mTOR inhibitors, $n(\%)$ & & $16(3.9)$ & $4(3.3)$ & $12(4.2)$ & \\
\hline $\begin{array}{l}\text { eGFR } 6 \text { months, } \mathrm{mL} / \mathrm{min} / 1.73 \mathrm{~m}^{2} \text {, } \\
\text { mean } \pm \mathrm{SD}\end{array}$ & $402(96.6)$ & $56.3 \pm 24.7$ & $54.6 \pm 25.5$ & $57 \pm 24.3$ & 0.38 \\
\hline Weight, mean \pm SD & $408(98.1)$ & $72.9 \pm 17.9$ & $72.3 \pm 17.4$ & $73.1 \pm 17$ & 0.69 \\
\hline $\mathrm{BMI}, \mathrm{kg} / \mathrm{m}^{2}$, mean $\pm \mathrm{SD}$ & $278(66.8)$ & $26.2 \pm 5.5$ & $26 \pm 6.3$ & $26.3 \pm 5.2$ & 0.7 \\
\hline
\end{tabular}

HD, heart disease; HTN, hypertension; PRA, panel reactive antibodies; DGF, delayed graft function; IL, interleukin; CCB, calcium channel blockers; ACE, angiotensin converting enzyme inhibitors; ARB, angiotensin receptor blockers; mTOR, mammalian target of rapamycin; eGFR, estimated glomerular filtration rate; BMI, body mass index.

For missing data analysis, we used multiple imputations with 5 repeats. All available variables were included and multiple logistic regression was used to impute missing variables.

For survival analysis, we used univariate and multivariate Cox proportional hazard model. As AKI event s occur over time, the analysis was done in time varying manner with AKI event as the time-dependent variable. The proportionality of hazard assumption was evaluated by examining the interaction between each variable and time. For estimated glomerular filtration rate (eGFR), the proportional hazard assumption was refuted. As a result, we evaluated the effect of eGFR quartiles on the primary outcome and found that only the lower quartile had significant effect on the primary outcome. As a result, to adjust for possible confounding effect of eGFR we stratified all multivariate analyses according to eGFR quartiles (the lowest quartile in 1 strata and the 3 other quartiles in the other). For multivariate analysis, we used stepwise forward regression model with a significance level of 0.05 for inclusion. To the results of the regression model, we added the factors that were significantly different between the groups and factors considered as possible confounders. The final model included age, history of heart disease, diabetes mellitus at 6 months, hypertension at 6 months, donor type (living or deceased), current smoking, treatment with statins, treatment with beta blockers, delayed graft function, and hospital stay after the transplantation procedure. 
Table 2. Association between AKI etiology and the main outcome

\begin{tabular}{|c|c|c|c|c|c|c|c|}
\hline \multirow[t]{2}{*}{ Etiology } & \multirow[t]{2}{*}{$n(\%)$} & \multicolumn{3}{|c|}{ Univariate } & \multicolumn{3}{|c|}{ Multivariate } \\
\hline & & HR & $95 \% \mathrm{CI}$ & $p$ value & HR & $95 \%$ CI & $p$ value \\
\hline Undetermined & $26(21.0)$ & 2.773 & $1.485-5.178$ & 0.001 & 2.712 & $1.4-5.252$ & 0.003 \\
\hline Acute rejection* & $7(5.6)$ & - & - & - & - & - & - \\
\hline Infection & $36(29.0)$ & 1.524 & $0.793-2.926$ & 0.206 & 1.784 & $0.897-3.551$ & 0.099 \\
\hline Medication toxicity & $14(11.3)$ & 2.600 & $1.206-5.606$ & 0.015 & 3.331 & $1.493-7.433$ & 0.003 \\
\hline Hypovolemia & $20(16.1)$ & 0.720 & $0.227-2.279$ & 0.576 & 0.303 & $0.086-1.066$ & 0.063 \\
\hline Others & $21(16.9)$ & 3.011 & $1.613-5.618$ & 0.001 & 2.031 & $1.032-3.998$ & 0.04 \\
\hline
\end{tabular}

* No events, HR could not be evaluated.

HR, hazard ratio; AKI, acute kidney injury.

Table 3. Association between AKI and the different components of the main outcome

\begin{tabular}{lllll}
\hline Outcome & $n$ & HR & $95 \%$ CI & $p$ value \\
\hline CAD & 48 & 1.37 & $0.73-2.56$ & 0.33 \\
CHF & 42 & 1.67 & $0.87-3.2$ & 0.15 \\
CVA & 23 & 1.85 & $0.77-4.44$ & 0.17 \\
PVD & 29 & 1.69 & $0.76-3.76$ & 0.2 \\
CV death & 26 & 4.23 & $1.9-10.33$ & 0.001 \\
All-cause death & 76 & 2.35 & $1.47-3.75$ & $<0.001$ \\
\hline
\end{tabular}

$\mathrm{HR}$, hazard ratio; CAD, coronary artery disease; CHF, congestive heart failure; CVA, cerebrovascular accident; PVD, peripheral vascular disease; CV, cardiovascular; AKI, acute kidney injury.

\section{Results}

Four hundred and fifty-eight patients underwent kidney transplantation in our center during the study period. Of them, 416 where included in the study. The reasons for exclusion are presented in Figure 1. The characteristics of patients included in the study are presented in Table 1.

One hundred and twenty-four patients (29.8\%) had a least 1 episode of AKI at a median time from transplantation of 266 days (IQR 60-760) and 12 patients (9.7\%) required renal replacement therapy. Patients with AKI at any time were significantly more likely to be current smokers and experienced delayed graft function after the transplantation. There was also a trend for patients with AKI to be hypertensive. The characteristics of patients who had AKI events during the follow-up are presented in Table 1.

The most prevalent etiologies for AKI were acute infection (36 patients), undetermined cause (26 patients), and hypovolemia (20 patients). The etiologies of all first AKI events are presented in Table 2 .

\section{Association of AKI and Primary Outcome: CV Including All-Cause Mortality}

During a median follow-up time of 7.2 years (IQR 4.3-9.1), 244 outcome events occurred in 144 patients; the outcome events are presented in Table 3. By time-varying Cox regression analysis, AKI was associated with an increased rate of CV outcome or death (hazard ratio [HR] $1.96,95 \%$ CI 1.36-2.81, $p<0.001$ ), and the association was still significant by multivariate model (HR 1.76, 95\% CI 1.18-2.63, $p=0.005$ ). 
Table 4. Subgroup analysis of the association between AKI and MACE including all-cause morbidity

\begin{tabular}{|c|c|c|c|c|}
\hline Subgroup & $n$ & HR & $95 \%$ CI & $p$ value \\
\hline \multicolumn{5}{|l|}{ Gender } \\
\hline Men & 267 & 2.25 & $1.49-3.41$ & $<0.001$ \\
\hline Women & 149 & 1.43 & $0.67-3.07$ & 0.36 \\
\hline \multicolumn{5}{|l|}{ Age, years } \\
\hline$<50$ & 202 & 2.66 & $1.24-5.69$ & 0.012 \\
\hline$>50$ & 214 & 2.03 & $1.34-3.07$ & 0.001 \\
\hline \multicolumn{5}{|c|}{ History of HD } \\
\hline No & 334 & 1.64 & $1.02-2.64$ & 0.04 \\
\hline Yes & 82 & 2.18 & $1.21-3.9$ & 0.009 \\
\hline \multicolumn{5}{|l|}{ Diabetes } \\
\hline No & 304 & 1.83 & $1.1-3.05$ & 0.021 \\
\hline Yes & 112 & 2.18 & $1.3-3.66$ & 0.03 \\
\hline \multicolumn{5}{|l|}{ HTN } \\
\hline No & 82 & 3.13 & $1.11-8.82$ & 0.031 \\
\hline Yes & 226 & 1.75 & $1.14-2.67$ & 0.01 \\
\hline \multicolumn{5}{|l|}{ Living donor } \\
\hline Yes & 221 & 1.85 & $1-3.41$ & 0.05 \\
\hline No & 195 & 1.88 & $1.19-2.95$ & 0.007 \\
\hline \multicolumn{5}{|l|}{ DGF } \\
\hline Yes & 107 & 1.72 & $0.98-3.03$ & 0.057 \\
\hline No & 296 & 1.67 & $1.01-2.85$ & 0.047 \\
\hline
\end{tabular}

Table 2 shows the association between the different etiologies of AKI and the primary outcome. Hypovolemia was not associated with an increased risk of the primary outcome, and by multivariate analysis there was a non-significant trend towards association with reduced risk. AKI due to infections was not associated with the primary outcome, however, by multivariate analysis there was a non-significant trend towards increased harm. Due to the low number of cases and no outcome events, the association between acute rejection and the primary outcome could not be evaluated.

Subgroup analysis for the main outcome of CV events and mortality is presented in Table 3. There was a significant interaction between gender and the association between AKI and cardiovascular disease (CVD; $p=0.011$ for interaction). While there was no association between AKI and CVD in women, in men it was associated with a highly increased risk. Other factors did not have an effect on the association between AKI and CVD.

\section{Association of AKI and Secondary Outcome: CV without All-Cause Mortality}

During the follow-up, 118 patients had an event of death censored CVD events (including $\mathrm{CV}$ death but censoring death from other causes). By univariate time-varying Cox analysis, AKI was associated with CV outcomes by univariate (HR 1.74, 95\% CI 1.17-2.58, $p=0.006$ ) and multivariate adjusted analysis (HR 1.8, 95\% CI 1.17-2.86, $p=0.008$ ), respectively.

\section{Association of AKI and Secondary Outcomes: CAD, CVA, PVD, CV Mortality and All-Cause Mortality}

Table 4 presents the association of AKI with the different components of the primary outcome. As can be seen, there was a trend for association of AKI with a worse outcome for all these outcomes. However, all-cause mortality and CV mortality were the only outcomes that were significantly associated with AKI. 
Rahamimov et al.: AKI and CV Events after Kidney Transplantation

Table 5. Association between AKI and MACE including all-cause morbidity, according to AKI timing

\begin{tabular}{|c|c|c|c|c|c|c|}
\hline \multirow[t]{2}{*}{ Timing } & \multicolumn{3}{|c|}{ Univariate } & \multicolumn{3}{|c|}{ Multivariate } \\
\hline & HR & $95 \% \mathrm{CI}$ & $p$ value & HR & $95 \%$ CI & $p$ value \\
\hline Less than 3 months & 2.01 & $1.2-3.36$ & 0.008 & 1.51 & $0.86-2.83$ & 0.12 \\
\hline Between 3 months and 1 year & 1.79 & $1-3.19$ & 0.049 & 2.02 & $1.09-3.72$ & 0.025 \\
\hline More than 1 year & 2.1 & $1.14-3.86$ & 0.017 & 1.98 & $1.03-3.81$ & 0.04 \\
\hline
\end{tabular}

MACE, major adverse cardiovascular events; AKI, acute kidney injury; HR, hazard ratio.

Association of AKI Timing and Primary Outcome: CV Including All-Cause Mortality

To evaluate the possible interaction between AKI timing and the risk of CVD, we analyzed the outcome data according to AKI timing post transplantation (within 3 months, between 3 months and 1 year, and more than 1 year). As can be seen in Table 5, there was no interaction between the timing of AKI and the primary outcome by univariate and multivariate Cox analyses. Although, by multivariate analysis AKI within the first 3 months was not associated with the risk of CVD, there was no difference compared to AKI events that occurred at other time intervals $(p>0.5)$.

Effect of Kidney Function Return to Baseline Value on the Association of AKI and Primary Outcome: CV Including All-Cause Mortality

Of the 118 AKI events during the follow-up period, the eGFR went down in 68 events (57.6\%) within a month to a value within $20 \%$ of baseline. These events were defined as fully recovered AKI. In contrast, AKI events in which the eGFR was more than $20 \%$ above baseline at 1 month were considered as non-recovered. By univariate time-varying Cox analysis, both recovered $\mathrm{AKI}$ and non-recovered $\mathrm{AKI}$ were associated with an increased rate of the primary outcome (HR 1.87, 95\% CI 1.2-2.7, $p=0.006$ ) and (HR 2.11, 95\% CI 1.26-3.53, $p=0.008$ ) respectively. However, by multivariate analysis, recovered AKI was not significantly associated with the primary outcome (HR 1.48, 95\% CI 0.9-2.44, $p=0.126$ ). Non-recovered AKI was still associated with the primary outcome even in the multivariate-adjusted model (HR $2.25,95 \%$ CI 1.3-3.9, $p=0.004$ ).

\section{Discussion}

In this study, we found a significant association between AKI events and MACEs in kidney transplant recipients. This association has not been described before in this group of patients.

This association was extensively studied in other populations: patients that developed AKI during hospitalization [13], patients after acute coronary syndrome [14] and percutaneous coronary interventions or bypass grafting [15, 16], tricuspid annuloplasty [17] or patients after non-cardiac operation $[18,19]$ with conflicting results [13]. In a recent metaanalysis that included 25 studies involving 254,408 adults [12], AKI was associated with an $86 \%$ increased risk of CV mortality and a 38\% increased risk of MACEs. When stratifying outcomes, a $58 \%$ increase in CHF with a positive correlation with AKI severity was found, and a $40 \%$ increase in acute myocardial infarction and a $15 \%$ increased rate of stroke were also found. In the present study, we found a significant increased risk for all-cause mortality and CV mortality in the kidney transplant patient population but not for the different components of the primary outcome. This is most probably due the relatively small sample size. Interestingly, AKI due to hypovolemia was not associated with MACE. It should be noted that in a prior 


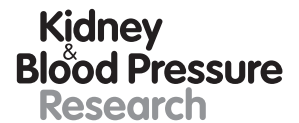

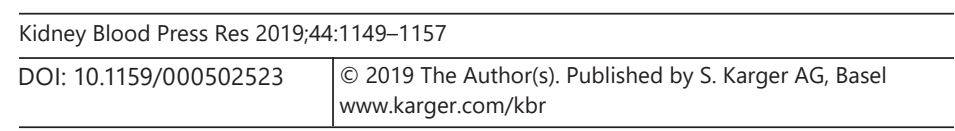

Rahamimov et al.: AKI and CV Events after Kidney Transplantation

study from our group, AKI due to hypovolemia was the only etiology not associated with reduced graft survival [1]. We can assume that the lack of structural kidney damage might be an important factor in these phenomena.

The positive association between AKI events and MACE does not necessarily mean causality as patients that are prone to develop AKI may have risk factors for CV events that might mediate the association [12]. It is interesting that non-recovered AKI was associated with an increased risk for the primary outcome. This finding correlates with studies in the non-transplant population that have found that in patients with hospital acquired AKI, complete renal recovery was associated with a lower risk of long-term MACE in comparison with those who did not fully recover [20]. Furthermore, non-recovery from dialysis was associated with a higher risk of CHF and death [21]. This suggests that the higher risk of MACE associated with AKI might be partly mediated by the known correlation between CKD and CV risk [22].

What distinguishes our research from other studies in different populations is the fact that kidney transplant recipients are a special combination of a transplanted kidney and a recipient that, up to the transplantation, do not share a common medical background or risk factors. As a result, the increased CVD risk associated with AKI cannot be attributed solely to risk factors in the recipient and the increased CV risk is more likely associated with the AKI events and not only with the propensity to develop AKI.

The association between AKI and MACE was found in our cohort to be positive only in male patients. This could be explained by the higher CV event rates in male transplant patients [23] and a reduced power to detect the effect of AKI in the subgroup of female patients due to a relatively small number of AKI events. We could not find any plausible explanation nor similar results in other populations to explain this difference [1-3].

Although our study represents a large contemporary cohort with sensitive methods for AKI and MACE identification and a sufficient follow-up time, it has several limitations. First, our study is a single-center, retrospective cohort study, which make its external validity somewhat limited. It is also important to note that our study population is all Caucasian, so our results may not be applicable for non-Caucasian populations. Second, we choose to use only one of the KDIGO laboratory criteria for the definition of AKI (elevation of SCr of 50\%) and not the additional criteria of absolute elevation of $0.3 \mathrm{mg} / \mathrm{dL}$ in SCr. We excluded these criteria since patients after kidney transplantation have a heterogeneous range of baseline $\mathrm{SCr}$ values and an absolute elevation of $0.3 \mathrm{mg} / \mathrm{dL}$ might be too sensitive. The result might be an over-diagnosis of AKI as $0.3 \mathrm{mg} / \mathrm{dL}$ might represent a relatively small percentage of the baseline creatinine value. However, it is possible that by using the more stringent definition of AKI some mild events were also missed. To prevent the effect of the donor factors and the perioperative instability, we excluded delayed graft function events and AKI events in the first 2 weeks post transplantation from analysis such that our results are not applicable for events occurring at this early period.

In conclusion, our results demonstrate a strong association between AKI events and the risk for CVD and death in patients after kidney transplantation. These findings may suggest that we should increase our awareness on CV risk prevention in patients with previous AKI events and consider the use of preventive measures, as well as encourage lifestyle modifications. As prospective studies cannot be done, further studies in larger and more heterogeneous populations are needed to validate our results.

\section{Disclosure Statement}

The authors declare no conflict of interest. 


\section{Kidney \\ Blood Pressure \\ Research}

\begin{tabular}{l|l}
\hline Kidney Blood Press Res 2019;44:1149-1157 \\
\hline DOI: 10.1159/000502523 & $\begin{array}{l}\text { @ 2019 The Author(s). Published by S. Karger AG, Basel } \\
\text { www.karger.com/kbr }\end{array}$ \\
\hline
\end{tabular}

Rahamimov et al.: AKI and CV Events after Kidney Transplantation

\section{References}

1 van Dijk TY, Rahamimov R, Chagnac A, van Dijk DJ, Mor E, Shlomai A, et al. The effect of cause, timing, kidney function recovery, and recurrent events on the prognosis of acute kidney injury in kidney transplant recipients. Clin Transplant. 2018 Oct;32(10):e13398.

2 Panek R, Tennankore KK, Kiberd BA. Incidence, etiology, and significance of acute kidney injury in the early post-kidney transplant period. Clin Transplant. 2016 Jan;30(1):66-70.

3 Mehrotra A, Rose C, Pannu N, Gill J, Tonelli M, Gill JS. Incidence and consequences of acute kidney injury in kidney transplant recipients. Am J Kidney Dis. 2012 Apr;59(4):558-65.

4 Filiponi TC, Requião-Moura LR, Tonato EJ, Carvalho de Matos AC, E Silva-Filho AP, de Souza Durão Junior M. Hospital Admission following Acute Kidney Injury in Kidney Transplant Recipients Is Associated with a Negative Impact on Graft Function after 1-Year. PLoS One. 2015 Sep;10(9):e0138944.

5 Nagarajan M, Ramanathan S, Dhanapriya J, Dineshkumar T, Subramaniyan TB, Gopalakrishnan N. Impact of acute kidney injury on renal allograft survival. Ren Fail. 2017 Nov;39(1):40-4.

6 Nakamura M, Seki G, Iwadoh K, Nakajima I, Fuchinoue S, Fujita T, et al. Acute kidney injury as defined by the RIFLE criteria is a risk factor for kidney transplant graft failure. Clin Transplant. 2012 Jul-Aug;26(4):520-8.

7 Abu Jawdeh BG, Govil A. Acute Kidney Injury in Transplant Setting: Differential Diagnosis and Impact on Health and Health Care. Adv Chronic Kidney Dis. 2017 Jul;24(4):228-32.

8 Molnar AO, van Walraven C, Fergusson D, Garg AX, Knoll G. Derivation of a Predictive Model for Graft Loss Following Acute Kidney Injury in Kidney Transplant Recipients. Can J Kidney Health Dis. 2017 Jan;4: 2054358116688228.

9 Rao NN, Coates PT. Cardiovascular Disease After Kidney Transplant. Semin Nephrol. 2018 May;38(3):291-7.

10 Soveri I, Holme I, Holdaas H, Budde K, Jardine AG, Fellström B. A cardiovascular risk calculator for renal transplant recipients. Transplantation. 2012 Jul;94(1):57-62.

11 Devine PA, Courtney AE, Maxwell AP. Cardiovascular risk in renal transplant recipients. J Nephrol. 2019 Jun; 32(3):389-399.

12 Odutayo A, Wong CX, Farkouh M, Altman DG, Hopewell S, Emdin CA, et al. AKI and Long-Term Risk for Cardiovascular Events and Mortality. J Am Soc Nephrol. 2017 Jan;28(1):377-87.

13 Omotoso BA, Abdel-Rahman EM, Xin W, Ma JZ, Scully KW, Arogundade FA, et al. Dialysis requirement, longterm major adverse cardiovascular events (MACE) and all-cause mortality in hospital acquired acute kidney injury (AKI): a propensity-matched cohort study. J Nephrol. 2016 Dec;29(6):847-55.

14 Otsuka K, Shimada K, Katayama H, Nakamura H, Ishikawa H, Takeda H, et al. Prognostic significance of renal dysfunction and its change pattern on outcomes in patients with acute coronary syndrome treated with emergent percutaneous coronary intervention. Heart Vessels. 2019 May;34(5):735-44.

15 Leistner DM, Münch C, Steiner J, Erbay A, Riedel M, Gebhard C, et al. Impact of acute kidney injury in elderly ( $\geq 80$ years) patients undergoing percutaneous coronary intervention. J Interv Cardiol. 2018 Dec;31(6): $792-8$

16 Warren J, Mehran R, Baber U, Xu K, Giacoppo D, Gersh BJ, et al. Incidence and impact of acute kidney injury in patients with acute coronary syndromes treated with coronary artery bypass grafting: Insights from the Harmonizing Outcomes With Revascularization and Stents in Acute Myocardial Infarction (HORIZONS-AMI) and Acute Catheterization and Urgent Intervention Triage Strategy (ACUITY) trials. Am Heart J. 2016 Jan; 171(1):40-7.

17 Wu MZ, Chen Y, Au WK, Chan D, Sit KY, Ho KL, et al. Predictive value of acute kidney injury for major adverse cardiovascular events following tricuspid annuloplasty: A comparison of three consensus criteria. J Cardiol. 2018 Sep;72(3):247-54.

18 Pourafkari L, Arora P, Porhomayon J, Dosluoglu HH, Arora P, Nader ND. Acute kidney injury after non-cardiovascular surgery: risk factors and impact on development of chronic kidney disease and long-term mortality. Curr Med Res Opin. 2018 Oct;34(10):1829-37.

19 Zhao XJ, Zhu FX, Li S, Zhang HB, An YZ. Acute kidney injury is an independent risk factor for myocardial injury after noncardiac surgery in critical patients. J Crit Care. 2017 Jun;39:225-31.

20 Omotoso BA, Abdel-Rahman EM, Xin W, Ma JZ, Scully KW, Arogundade FA, et al. Acute kidney injury (AKI) outcome, a predictor of long-term major adverse cardiovascular events (MACE). Clin Nephrol. 2016 Jan;85(1): $1-11$.

21 Lee BJ, Hsu CY, Parikh RV, Leong TK, Tan TC, Walia S, et al. Non-recovery from dialysis-requiring acute kidney injury and short-term mortality and cardiovascular risk: a cohort study. BMC Nephrol. 2018 Jun;19(1):134.

22 Di Lullo L, House A, Gorini A, Santoboni A, Russo D, Ronco C. Chronic kidney disease and cardiovascular complications. Heart Fail Rev. 2015 May;20(3):259-72.

23 Seoane-Pillado MT, Pita-Fernández S, Valdés-Cañedo F, Seijo-Bestilleiro R, Pértega-Díaz S, Fernández-Rivera $\mathrm{C}$, et al. Incidence of cardiovascular events and associated risk factors in kidney transplant patients: a competing risks survival analysis. BMC Cardiovasc Disord. 2017 Mar;17(1):72. 